\title{
El Mercado Verde en Latinoamérica y la Responsabilidad Social Empresarial
}

\author{
Sánchez David ${ }^{1}$ Aguilar Kevin ${ }^{2}$ \\ ORCID: https://orcid.org/0000-0003-3704-641911, ORCID: https://orcid.org/0000-0001-9932-2584² \\ david.sanchez@udla.edu.ec ${ }^{1}$, kevin.aguilar@udla.edu.ec ${ }^{2}$ \\ Universidad de las Américas \\ Quito-Ecuador
}

Recibido (01/07/20), Aceptado (17/07/20)

\begin{abstract}
Resumen: El presente trabajo se enfocó a profundizar sobre el tema de las economías verdes y la Responsabilidad Social Empresarial. A través de una investigación documental, se logró evidenciar que las economías verdes siguen estando muy poco desarrolladas en todas partes del mundo, donde Europa es la que más avances posee en dicho tema, quedándose Latinoamérica rezagada al respecto por problemas tecnológicos y de desarrollo. Sobre la responsabilidad social se pudo entender que la misma representa una piedra angular para el desarrollo de las naciones y una de las vías más relevantes para alcanzar la sostenibilidad, reduciéndose problemas como la pobreza y el impacto medioambiental. Se concluyó que la importancia de estos trabajos de investigación radica en la amplitud del análisis que proponen, lo que sirve como un elemento referencial de gran calado desde el cual partir para otras investigaciones.
\end{abstract}

Palabras Clave: Sostenibilidad, Responsabilidad, Sociedad, Desarrollo.

\section{The Green Market in Latin America and Social Responsibility Business}

\begin{abstract}
This work focused on deepening on the topic of green economies and Corporate Social Responsibility. Through a documentary investigation, it was possible to show that green economies are still very underdeveloped in all parts of the world, where Europe is the one that has the most progress on this issue, with Latin America lagging behind in this regard due to technological and development problems. Regarding social responsibility, it was understood that it represents a cornerstone for the development of nations and one of the most relevant ways to achieve sustainability, reducing problems such as poverty and environmental impact. It was concluded that the importance of these research works lies in the breadth of the analysis they propose, which serves as a referential element of great significance from which to start for other investigations.
\end{abstract}

Keywords: Sustainability, Responsibility, Society, Development. 


\section{I.INTRODUCCIÓN}

Los mercados verdes son una tendencia que muchas empresas alrededor del mundo están tomando en la actualidad. En Latinoamérica esto también es posible de observar. Ahora, el problema radica en evidenciar si el mercado en esta región estaría a la par de mercados verdes en zonas desarrolladas de Europa y Asia, así como en naciones africanas y la propia Estados Unidos. Entender sobre ello será la finalidad u objetivo general de este trabajo, a la par que también lo será, en una medida más amplia, la respuesta a una serie de preguntas relacionadas con la responsabilidad empresarial. El fundamento del estudio radica en la importancia de estos mercados verdes y el de la propia responsabilidad empresarial, todo de cara a una economía y desarrollo humano sostenibles. Para ello, se apoyaría el trabajo en una metodología de investigación documental a partir de fuentes relevantes que ayudasen a argumentar lo que acá se expondría.

El estudio se dividirá, junto a esta introducción, en dos partes más. Una sección de desarrollo en la cual se generará la discusión de los diferentes puntos relacionados a esta investigación y las respuestas a las siete preguntas de meta cognición que estarán embebidas en el cuerpo del mismo. Esto se hará en base a las fuentes bibliográficas consultadas al respecto. Por último, se ofrecerá una sección de conclusiones que, más allá de un resumen de los puntos tratados, se alejará de este fin y planteará la importancia del trabajo realizado, extensiones del mismo y potenciales aplicaciones a futuro. Todo ello a continuación sin más dilaciones.

\section{II.DESARROLLO}

En la región Latinoamericana los mercados verdes están siendo aún poco aprovechados. En algunos reportajes bastante recientes sobre el tema, se ha explicado que, en Latinoamérica, incluyendo Ecuador, se han generado avances para el desarrollo de economías sostenibles direccionadas al propósito verde, sobre todo en vinculación con la sociedad y la reducción de la pobreza [1]. El problema radica en que América Latina, por su bajo desarrollo tecnológico, está siendo mucho más lenta en aprovechar estas nuevas tendencias, lo cual reduce dramáticamente los impactos positivos de la misma en el corto plazo y genera incertidumbres sobre el tema en estas naciones. Por ello, pese a que Latinoamérica posee un gran potencial por tener una gran biodiversidad y muchos recursos naturales bastante accesibles [1], la economía verde no parece en este momento ser el centro de atención de las economías latinoamericanas y no parecerá que lo sean en el corto plazo.

Ahora, si se compara este hecho con las economías verdes en área como Europa, Estados Unidos, Asia y África, las diferencias no podrían considerarse tampoco abismales, pero en efecto, sí son importantes. Estados Unidos quizás es la nación de las desarrolladas que menos apuesta por la economía verde, siendo posible evidenciar una lentitud evidente en el desarrollo de sus tecnologías y transiciones productivas a esta nueva tendencia [2]. Europa, por su parte, tiene uno de los mercados verdes más desarrollados en la actualidad, apoyado por políticas económicas de gran calado para evitar que se genere un deterioro más importante en el medio ambiente. Pese a ello, muchos sectores en Europa siguen esperando ser atendidos en relación a la economía verde, pero el camino ya se ha trazado y parece cuestión de tiempo para que lo anterior llegue.

En África y Asia la situación es distinta. En la región africana las naciones están palpablemente atrasados en los aprovechamientos de los mercados verdes, incluso más que en América Latina, debido a la pobreza de la región [2]. No se puede considerar que África tendría una economía verde fortalecida sólo por naciones como Sudáfrica, dado que en realidad las demás naciones, como la República del Congo u otras, están explotando sus minas y tierras de forma totalmente alejada de la responsabilidad empresarial que debería existir en base a las economías verdes.

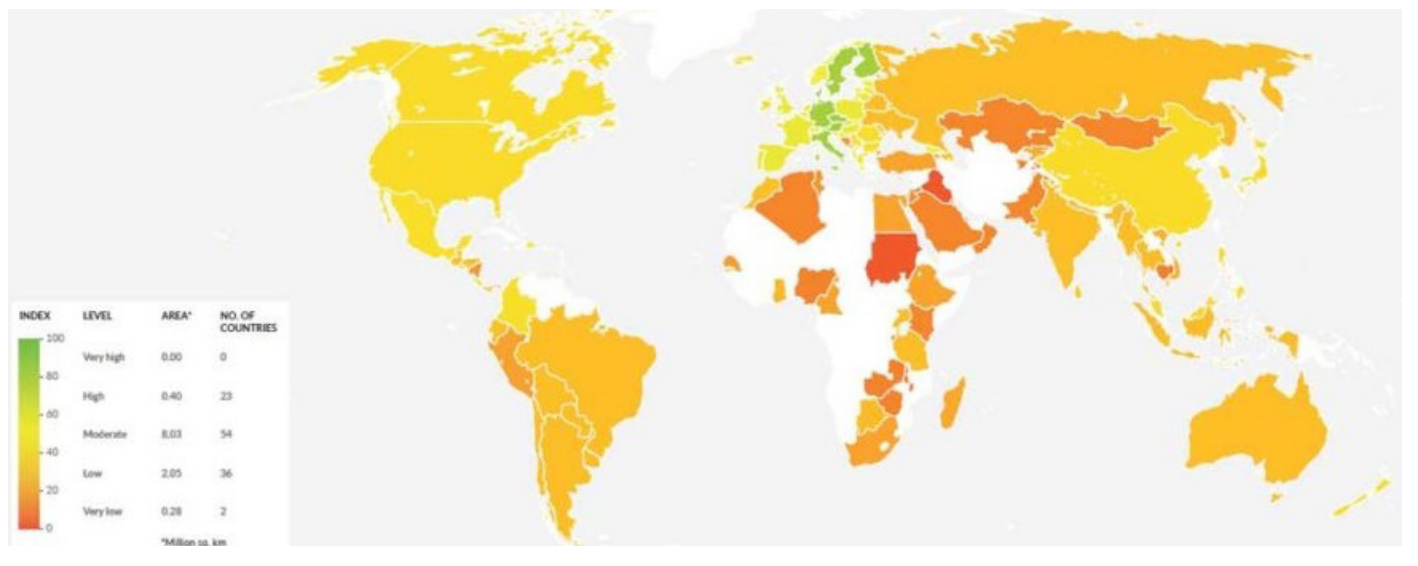

Figura 1. Mapa del índice de crecimiento económico verde en las naciones de todo el globo. Fuente: GGGI [3].

Sobre Asia la realidad no se aleja mucho de lo expuesto en Estados Unidos. Ciertamente, dado el grado de desarrollo de muchas naciones asiáticas, la región está avanzando correctamente hacia la ruta de la sostenibilidad, en especial en el aprovechamiento de las tecnologías para generar nuevas formas de producción sostenibles a lo largo del tiempo y mejorar las situaciones de empleo a través de ellas [2]. El problema para Asia es que existen muchas naciones que, pese a su volumen productivo y su palpable crecimiento económico, han generado muy pocas inversiones en el aspecto de las economías verdes, 
lo que reduce el impacto general de esta región en base a esta nueva tendencia. Eso sí, tanto para Asia como para África, el incremento del trabajo agrícola se paraleliza con un mejoramiento de las condiciones de vida de las personas, por lo que, de cara al futuro, la agricultura y la ganadería, responsables desde el aspecto verde, podrían ser puntos fuertes para el desarrollo económico sostenible para estas naciones del oriente.

En la Figura 1 se puede comprobar todo lo mencionado mediante una gráfica del desarrollo de las economías verdes en todas las naciones a nivel mundial. Por ello, es importante preguntarse sobre la posibilidad del impacto de la responsabilidad empresarial para acelerar un crecimiento de los mercados verdes que, a todas luces a partir de la gráfica señalada, están en términos generales muy atrasados y poco explotados. Esta situación, a la par de preocupante, genera incertidumbre acerca de las prioridades de los mercados en la actualidad. ¿El crecimiento económico estará siempre por encima de la sostenibilidad de la especie humana? Esta parece una pregunta que, en la actualidad, posee una respuesta positiva, pero que quizás con el paso del tiempo, la tendencia pueda revertirse de forma esperanzadora para los mercados verdes.

\section{A.¿Qué es una empresa socialmente responsable?}

Una empresa socialmente responsable es aquella que realiza sus actividades de producción y generación de beneficios entendiendo la necesidad de enmarcar todas ellas en la filosofía de la sustentabilidad, procurando que todas las acciones sean responsables con el medio ambiente, la sociedad y la economía en sí misma [4]. En este sentido, la responsabilidad social empresarial considera que las empresas deben comprender las necesidades de los actores de su entorno con la finalidad de reducir el impacto de las mencionadas acciones en el corto plazo y el largo plazo. También se debe procurar que la empresa retribuya en beneficios de diversos tipos a la comunidad, el ambiente e incluso la propia economía, por los beneficios que haya podido obtener de sí.

En este sentido, una empresa socialmente responsable es una empresa que entiende la necesidad de proyectar sus actividades hacia el futuro, mejorándose continuamente para que su impacto positivo sea siempre sustancialmente mayor que el negativo, uno que lamentablemente siempre existirá pero que se debe procurar reducir al máximo [4]. Las empresas socialmente responsables son una tendencia bastante importante en la actualidad, debido a que gracias a las mismas se puede perfilar el mundo hacia el desarrollo donde seres humanos, sociedad y medio ambienten encuentren puntos en común para crecer conjuntamente sin que uno se vea más perjudicado por el otro. Este equilibrio es complicado de conseguir, pero los beneficios de este son inexpugnables y representan un hito que se persigue con asiduo en la actualidad.

\section{B.¿Qué es la responsabilidad social?}

La responsabilidad social, evidentemente, está epistemológica y etimológicamente ligada a la responsabilidad social empresarial. La responsabilidad social puede comprenderse como aquel compromiso que se adquiere por una persona, ya sea persona jurídica o persona natural, para con los individuos o la comunidad dentro de una sociedad, con la finalidad de realizar acciones y tomar decisiones que afecten positivamente a las comunidades y les permitan aprovechar las oportunidades que esa misma persona ha tenido en relación con la actividad que realiza [5].

La responsabilidad social, pese a ser relativamente nueva en el ámbito empresarial, la realidad sugiere que es bastante antigua, apareciendo por primera vez el término en la antigua Grecia dentro de las reflexiones de varios filósofos de la época en relación con la necesidad que muchos comerciantes tenían con la sociedad que le permitía obtener beneficios a costa de su trabajo y colaboración, directa o indirecta. Para los griegos, esta realidad de responsabilidad pasaba por el hecho de generar una estabilidad social en donde las personas pudieran comprender que lo que una persona obtenía, cuando era desproporcionado en relación con lo que los demás poseían, tuviese un punto de inflexión social otorgándole un beneficio a los demás a raíz de esta situación.

\section{C.¿Cuál es el rol del ingeniero en la responsabilidad social industrial?}

Por supuesto, para lograr la responsabilidad social empresarial, así como la industrial, se debe contar con profesionales que ayuden a encaminar las actividades de las empresas hacia estos fines. En este sentido, la responsabilidad social no es sólo un acto de la empresa, sino que debe ser el reflejo de la actuación de sus empleados con relación a esta finalidad [4]. Así, el rol del ingeniero en la responsabilidad social industrial es el de generar mecanismos, desde su trinchera de actuación, para asegurar que las actividades a las que está al mando generan el menor impacto posible a la sociedad y que, por el contrario, son capaces de relacionarse al tratamiento de algunas necesidades de la comunidad.

De tal forma, un ingeniero podría tener un rol vital en, por ejemplo, reducir el impacto ambiental de un proceso o incluso optimizarlo de tal forma que se pueda generar mayor producto a menores costos para hacer del mismo más accesible para la comunidad. En este sentido, el rol del ingeniero dentro de la responsabilidad social empresarial es ampliamente variado. En este sentido, para lograr esta responsabilidad, el ingeniero debe introducir su rol dentro de los principios de innovación, transparencia, eficiencia, confianza y legitimidad para generar empresas más competitivas en el largo plazo y un desarrollo sustentable en las mismas [6].

\section{D.¿De qué manera se pueden hacer empresas socialmente responsables?}

Para conseguir empresas socialmente responsables se pueden hacer varias cosas. Por un lado, se debe generar una estrategia de sostenibilidad para la empresa, con políticas y procedimientos claros para estos fines, de tal forma que las acciones de la empresa puedan asociarse a la responsabilidad social deseada [4]. También se debe generar una transformación en los procesos de compra y venta de productos para reducir los impactos que se pueden estar generando por estas acciones. El apoyo a proyectos de tipo social y ambiental, así como la incorporación de políticas de Responsabilidad Social Empresarial 
por departamento puede ser muy beneficiosa para estos fines [4]. Finalmente, la empresa debe generar metas sostenibles al largo plazo que realmente se asocien a las necesidades de su entorno para que las acciones sean acordes al beneficio social.

\section{E.¿Qué empresas conoce usted que cumplan con la responsabilidad social empresarial?}

Estas estrategias anteriormente mencionadas pueden ser observadas en algunas empresas ecuatorianas, como bien podría ser Nestlé Ecuador o la Corporación Favorita. Estas empresas no sólo están transformando progresivamente sus procesos hacia la economía verde, sino que además genera proyectos sostenibles en donde buscan beneficiar al medio ambiente (siembra de árboles, concientización de las personas, entre otros) así como a las personas de ciertas comunidades claramente desfavorecidas, generando incluso fuentes de empleo para ellas.

\section{F.De tener usted una empresa, ¿qué tipo de responsabilidad social le gustaría implementar?}

En el caso que se tuviese una empresa, la responsabilidad social empresarial que sería ideal aplicar es aquella que se enfoca al desarrollo comunitario a través de las actividades de la empresa. Otorgar oportunidades de crecimiento a las personas es excelente, por lo que abrir espacios para que puedan obtener ingresos, puedan aprender cosas o mejorar habilidades hacia un cierto oficio, sería una forma de impactar positivamente en la comunidad y lograr que las personas en ella logren mejorar su perspectiva hacia el futuro.

\section{G.¿Qué factores influyen en el incumplimiento de la responsabilidad social?}

Lamentablemente, todo lo mencionado sobre la Responsabilidad Social Empresarial puede ser afectado por diversos factores. Las empresas, al ser parte de un sistema macro, pueden verse sometidas a muchas variables que pueden afectar el cumplimiento de la responsabilidad social empresarial. Uno de los más evidentes es el rubro económico donde la empresa labora, siendo más complicado de cumplir si no se asocia a una necesidad de la comunidad donde se ubica el negocio. Otro problema puede ser la filosofía de los accionistas o dueños de la empresa, quienes pueden ver a la responsabilidad social empresarial como algo innecesario. La falta de compromiso de la alta gerencia también puede afectar a que la responsabilidad social no se cumpla. Factores culturales pueden también afectar en la forma como participan las comunidades en los actos de responsabilidad social de la empresa, afectando los beneficios que puede aportar la misma.

\section{III.CONCLUSIONES}

A consecuencia de lo expuesto se puede concluir que la responsabilidad social empresarial es muy relevante no sólo para las comunidades o el medio ambiente, sino para el desarrollo y supervivencia a largo plazo de los seres humanos. Las actividades empresariales históricamente han generado impactos negativos en el medio ambiente y la sociedad, por lo que gracias a la Responsabilidad Social Empresarial esta situación se busca reducir de forma evidente. La importancia de la responsabilidad social empresarial, por ende, pasa por el hecho de ser una puerta única de conciliación entre el avance del ser humano y el respeto por el medio ambiente y la lucha por la reducción de la pobreza, problemas prevalentes y de gran calado en la actualidad en todo el planeta.

Este completo trabajo, aparte, sirvió para entender de forma más profunda sobre la responsabilidad social empresarial, por lo que su funcionalidad como elemento contextual del tema es más que claro. Así, podría aplicarse el uso del mismo como parte de las referencias de un marco teórico para algún trabajo de titulación o incluso servir para fundamentar las bases de una investigación o un proyecto de aplicación para la responsabilidad social de una empresa. Por todo ello, se sugiere que esta clase de trabajos se extiendan en el tiempo, dado que otorgan una gran utilidad para la comunidad académica y científica a la vez.

\section{REFERENCIAS}

[1]M. Mordt, «Soluciones ambientales sostenibles y economía verde e inclusiva: América Latina en la senda del cambio,» 2019.

[2]A. Martínez y A. Porcelli, «Reflexiones sobre la economía verde. El New Deal Ecológico Mundial,» Revista LEX, vol. 15, n 19 , pp. 361-408, 2017.

[3] Global Green Growth Institute, «Green Growth Index Concept,» 2019.

[4]J. F. Cajiga Calderón, «El concepto de responsabilidad social empresarial,» Ciudad de México, 2007.

[5]J. A. Cortés y E. Flores, Responsabilidad social, Centro de Investigación y Desarrollo Ecuador, 2017.

[6]J. M. Araque Ramírez, «Responsabilidad Social del Ingeniero,» San José de Cúcuta, 2016. 


\section{RESUMEN CURRICULAR}

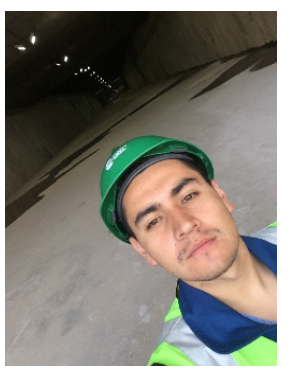

Kevin Aguilar, Estudiante de la carrera de Ingeniería Industrial de la Universidad de las Américas (UDLA).

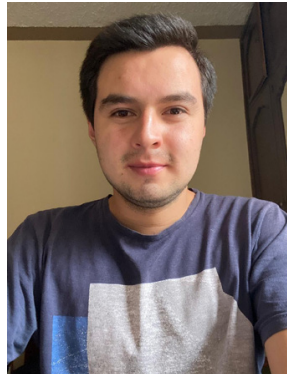

David Sánchez, Estudiante de la carrera de Ingeniería Industrial de la Universidad delas Américas (UDLA). 